\title{
钯催化的肉桂基碳酸酯化合物与酰腙的烯丙基胺化反应
}

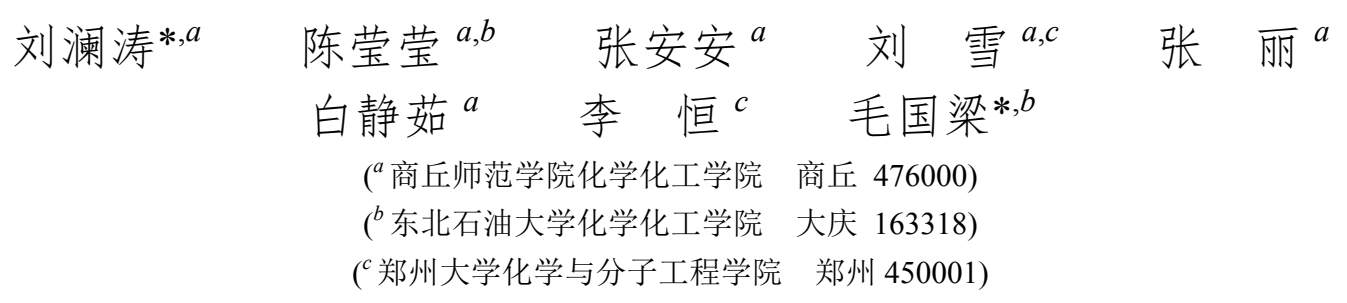

\begin{abstract}
摘要 烯丙基胺结构单元广泛存在于天然产物、药物和功能材料分子中, 另外烯丙基胺衍生物也是一类重要的有机合 成子. 实现了钯催化的肉桂基碳酸酯化合物和酰腙化合物的烯丙基胺化反应，高选择性地生成了线型烯丙基胺类化合 物, 产率高达 $99 \%$. 该方法具有不需要加碱、条件温和、操作简单、底物适应范围广等优点. 关键词＼cjkstart钯催化剂; 烯丙基胺化反应; 肉桂基碳酸酯
\end{abstract}

\section{Palladium Catalyzed Allylic Amination of Cinnamyl Carbonates with Acyl Hydrazones}
Liu, Lantao ${ }^{*, a}$
Chen, Yingying ${ }^{a, b}$
Zhang, $\mathrm{An}^{\prime} \mathrm{an}^{a}$
Liu, Xue $\mathrm{X}^{a, c}$
Zhang, $\mathrm{Li}^{a}$
Bai, Jingru $^{a}$
Li, Heng ${ }^{c}$
Mao, Guoliang ${ }^{*, b}$

$\left({ }^{a}\right.$ College of Chemistry and Chemical Engineering, Shangqiu Normal University, Shangqiu 476000)

$\left({ }^{b}\right.$ College of Chemistry and Chemical Engineering, Northeast Petroleum University, Daqing 163318)

( ${ }^{c}$ College of Chemistry and Molecular Engineering, Zhengzhou University, Zhengzhou 450001)

\begin{abstract}
Allylic amines moiety exists extensively in natural products, medicines and functional materials. In addition, they are also a kind of versatile building blocks for organic synthesis. Using $\mathrm{CH}_{3} \mathrm{CN}$ as solvent, the palladium catalyzed allyl amination of cinnamyl carbonate and acylhydrazone compounds was realized under argon. The linear product was formed selectively and the up to $99 \%$ yield was obtained. The reaction has features of base free, mild reaction condition, simple operation and broad substrate scope.

Keywords paladium catalysis; allylic amination; cinnamyl carbonate
\end{abstract}

含氮化合物在药物和功能材料等领域有着广泛的 应用, 因此它们的合成方法研究引起了化学家们极大的 兴趣 ${ }^{[1]}$. 含烯丙胺结构单元的化合物广泛存在于具有生 物活性的天然产物中 ${ }^{[2]}$. 部分烯丙基胺类化合物, 如外 用局部抗真菌药物菜替芬 ${ }^{[3]}$ 及口服抗真菌药物特比荎 芬 ${ }^{[4]}$, 已经发展成为高效、低毒的抗真菌药物(图 1). 烯 丙胺类化合物是合成天然产物的重要中间体, 已成功地 被用于 rosephilin、士的宁和 $(+)-\gamma$-lycorane 类天然生物 碱的合成 ${ }^{[5]}$. 此外, 烯丙胺类化合物还可以用于吲哚 ${ }^{[6]}$ 、 喹啉等杂环化合物 ${ }^{[7]}$ 、氨基酸的合成 ${ }^{[8]}$ 以及树脂等材料
的制备 ${ }^{[9]}$.

鉴于烯丙基胺类化合物的重要性，经过努力,化学 家们已经发展了过渡金属催化的亲核取代烯丙基胺化 反应 ${ }^{[10]} 、 \alpha, \beta$-不饱和酫与胺的还原烷基化反应 ${ }^{[1]]}$ 、亚胺

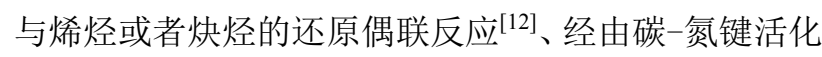
的未经活化的末端烯烃与二氨基甲烷衍生物的偶联反

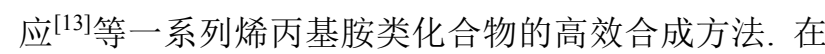
这几种合成策略中，过渡金属催化的亲核取代烯丙基 胺化反应取得了重要的进展. 钯催化的烯丙基醇、烯丙 基卤化物、烯丙基碳酸酯和烯丙基乙酸酯等烯丙基化

\footnotetext{
*Corresponding authors. E-mail: 1iult05@iccas.ac.cn; maoguoliang@nepu.edu.cn

Received August 13, 2018; revised September 3, 2018; published online September 10, 2018.

Project supported by the National Natural Science Foundation of China (No. 21572126), the Program for Science \& Technology Innovation Talents in Universities of Henan Province (No. 14HASTIT016) and the Program of Science and Technology Innovation Talents of Henan Province (No. 2018JQ0011). 国家自然科学基金(No. 21572126)、河南省高校科技创新人才基金(No. 14HASTIT016)和河南省科技创新杰出人才(No. 2018JQ0011)资助项目.
} 
合物的 $N$-烯丙基化反应已发展成为合成 $N$-烯丙胺类化 合物重要的方法之一, 在天然产物和氨基酸合成中具 有重要意义 ${ }^{[14]}$. 不同含氮化合物的笁选是从事该反应 研究的化学家们关注点之一, 多种含氮化合物, 如氨、 胺、叠氮化物、酰胺、酰亚胺, 已被成功应用于该反 应 ${ }^{[15]}$. 酰腙衍生物作为一类重要的含氮化合物, 其烯 丙基化反应并不常见. 2015 年王春江课题组 ${ }^{[16]}$ 成功实 现了有机小分子催化的酰腙衍生物的烯丙基化反应, 高对映选择性地得到了支链产物. 最近, 肖文精课题 组 ${ }^{[17]}$ 利用钯/亚磷酰胺配体催化体系, 首次实现了钯 催化酰腙衍生物的高对映选择性烯丙基化反应，高选 择性地得到了支链产物, $e e$ 值高达 $99 \%$. 本工作研究了 钯催化下肉桂基碳酸酯化合物与芳香醛衍生的酰腙化 合物的烯丙基化反应，高选择性地生成了一系列线型 烷基化产物.

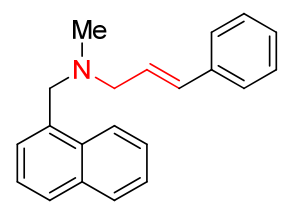

䒺替芬

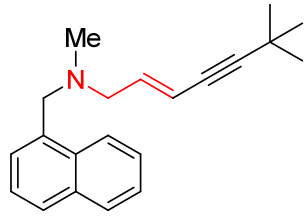

特比萗芬
图 1 含烯丙胺结构单元的抗真菌药物

Figure 1 Antifungal drugs containing allylic amine moiety

\section{1 结果与讨论}

\section{1 反应条件的篮选}

起初我们是在碱的存在下进行的研究, 虽然在此反 应条件下反应生成了目标产物 3aa. 但是经过多次实验 研究发现产物产率比较低, 同时还会有副产物肉桂醇生 成. 通过查阅文献, 将碱去掉, 产率有了提高 ${ }^{[18]}$, 然后 在不加碱的情况下, 进行了一系列的条件篮选. 在 $\mathrm{PPh}_{3}$ 和 $\operatorname{Pd}(\mathrm{OAc})_{2}$ 的催化下肉桂基甲基碳酸酯(1a)与 $N$-酰腙 化合物 2a 选择性地生成了线型产物 3aa(表 1, Entry 1). 接下来考察了配体、钯源、溶剂、反应温度、反应时间、 底物配比等因素对产物收率的影响，结果见表 1 .

配体篎选表明, 以 $\mathrm{Cy}_{3} \mathrm{P}$ 为配体时, 该反应不能发生 (表 1, Entry 2). rac-BINAP 能以 55\%的产率给出产物(表 1, Entry 3). 接着, 对钯源进行了篮选，使用 $\mathrm{Pd}(\mathrm{OAc})_{2}$ 时 产率最高(表 1, Entries 4 7 vs Entry 1). 溶剂的篎选中 (Entries 1,8 11) 发现, 溶剂为 $\mathrm{CH}_{3} \mathrm{CN}$ 时反应效果最好. 在确定了配体、钯源和溶剂后, 又对 $\mathrm{PPh}_{3}$ 与 $\mathrm{Pd}(\mathrm{OAc})_{2}$ 的配比(Entries 9，12，13)和底物 1a/2a 的配比(Entries $14 \sim 16)$ 进行了篮选. 最终发现当 $\mathrm{PPh}_{3}$ 的用量为 20 $\mathrm{mol} \%, \mathrm{Pd}(\mathrm{OAc})_{2}$ 的用量为 $5 \mathrm{~mol} \%$, 底物 $\mathbf{1 a} / \mathbf{2 a}$ 的物质的 量之比为 $1.5: 1$ 时产率达到了 $88 \%$ (表 1, Entry 15). 然
表 1 影响化合物 3aa 产率的因素 ${ }^{a}$

Table 1 Effects on the yields of compound 3aa

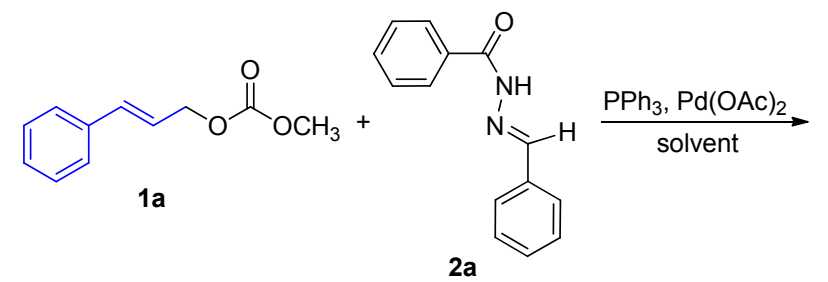

2a

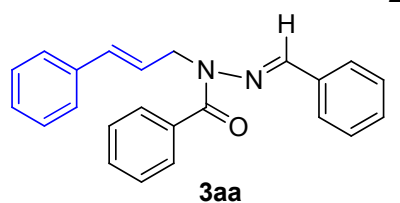

\begin{tabular}{clllc}
\hline Entry & \multicolumn{1}{c}{ Ligand } & \multicolumn{1}{c}{$[\mathrm{Pd}]$} & \multicolumn{1}{c}{ Solvent } & Yield $^{b} \%$ \\
\hline 1 & $\mathrm{PPh}_{3}$ & $\mathrm{Pd}(\mathrm{OAc})_{2}$ & Toluene & 63 \\
2 & $\mathrm{Cy}_{3} \mathrm{P}$ & $\mathrm{Pd}(\mathrm{OAc})_{2}$ & Toluene & 0 \\
3 & rac-BINAP $\mathrm{Pd}(\mathrm{OAc})_{2}$ & Toluene & 55 \\
4 & $\mathrm{PPh}_{3}$ & $\mathrm{Pd}_{2}(\mathrm{dba})_{3}$ & Toluene & 34 \\
5 & $\mathrm{PPh}_{3}$ & $\mathrm{Pd}(\mathrm{dba})_{2}$ & Toluene & 23 \\
6 & $\mathrm{PPh}_{3}$ & $\mathrm{PdCl}_{2}$ & Toluene & 21 \\
7 & $\mathrm{PPh}_{3}$ & $\mathrm{PdCl}\left(\mathrm{PPh}_{3}\right)_{2}$ & Toluene & 20 \\
8 & $\mathrm{PPh}_{3}$ & $\mathrm{Pd}(\mathrm{OAc})_{2}$ & $\mathrm{DCE}$ & 62 \\
$9^{c}$ & $\mathrm{PPh}_{3}$ & $\mathrm{Pd}(\mathrm{OAc})_{2}$ & $\mathrm{CH}_{3} \mathrm{CN}$ & 64 \\
10 & $\mathrm{PPh}_{3}$ & $\mathrm{Pd}(\mathrm{OAc})_{2}$ & $\mathrm{DMF}$ & 48 \\
11 & $\mathrm{PPh}_{3}$ & $\mathrm{Pd}(\mathrm{OAc})_{2}$ & $\mathrm{THF}$ & 20 \\
$12^{d}$ & $\mathrm{PPh}_{3}$ & $\mathrm{Pd}(\mathrm{OAc})_{2}$ & $\mathrm{CH}_{3} \mathrm{CN}$ & 70 \\
$13^{e}$ & $\mathrm{PPh}_{3}$ & $\mathrm{Pd}(\mathrm{OAc})_{2}$ & $\mathrm{CH}_{3} \mathrm{CN}$ & 75 \\
$14^{e, f}$ & $\mathrm{PPh}_{3}$ & $\mathrm{Pd}(\mathrm{OAc})_{2}$ & $\mathrm{CH}_{3} \mathrm{CN}$ & 84 \\
$15^{e, g}$ & $\mathrm{PPh}_{3}$ & $\mathrm{Pd}(\mathrm{OAc})_{2}$ & $\mathrm{CH}_{3} \mathrm{CN}$ & 88 \\
$16^{e, h}$ & $\mathrm{PPh}_{3}$ & $\mathrm{Pd}(\mathrm{OAc})_{2}$ & $\mathrm{CH}_{3} \mathrm{CN}$ & 88 \\
$17^{e, g}$ & $\mathrm{PPh}_{3}$ & $\mathrm{Pd}(\mathrm{OAc})_{2}$ & $\mathrm{CH}_{3} \mathrm{CN}\left(75{ }^{\circ} \mathrm{C}\right)$ & 74 \\
$18^{e, g}$ & $\mathrm{PPh}_{3}$ & $\mathrm{Pd}(\mathrm{OAc})_{2}$ & $\mathrm{CH}_{3} \mathrm{CN}\left(65{ }^{\circ} \mathrm{C}\right)$ & 67 \\
$19^{e, g}$ & $\mathrm{PPh}_{3}$ & $\mathrm{Pd}(\mathrm{OAc})_{2}$ & $\mathrm{CH}_{3} \mathrm{CN}(16 \mathrm{~h})$ & 65 \\
$20^{e, g}$ & $\mathrm{PPh}_{3}$ & $\mathrm{Pd}(\mathrm{OAc})_{2}$ & $\mathrm{CH}_{3} \mathrm{CN}(24 \mathrm{~h})$ & 99 \\
\hline
\end{tabular}

${ }^{a}$ Reaction condition: 1a $(0.1 \mathrm{mmol}), \mathbf{2 a}(0.1 \mathrm{mmol})$, ligand $(10 \mathrm{~mol} \%)$, [Pd] (5 mol\%), in solvent at $85{ }^{\circ} \mathrm{C}, 20 \mathrm{~h} .{ }^{b}$ Isolated yield. ${ }^{c} \mathrm{PPh}_{3}(10 \mathrm{~mol} \%), \mathrm{Pd}(\mathrm{OAc})_{2}$ (5 mol\%). ${ }^{d} \mathrm{PPh}_{3}$ (15 mol\%), $\mathrm{Pd}(\mathrm{OAc})_{2}(5 \mathrm{~mol} \%) .{ }^{e} \mathrm{PPh}_{3}(20 \mathrm{~mol} \%)$, $\mathrm{Pd}(\mathrm{OAc})_{2}(5 \mathrm{~mol} \%) .{ }^{f} n(\mathbf{1 a}): n(\mathbf{2 a})=1.2: 1 .{ }^{g} n(\mathbf{1 a}): n(\mathbf{2 a})=1.5: 1$. ${ }^{h} n(\mathbf{1 a}): n(\mathbf{2 a})=2: 1$.

后又对温度进行了篮选(Entries 15, 17, 18), 实验证明当 反应温度为 $85{ }^{\circ} \mathrm{C}$ 时产率最高. 最后对反应时间进行了 筛选(Entries 15, 19, 20), 实验发现将反应时间延长为 24 $\mathrm{h}$, 产率提高至 $99 \%$.

综上所述, 最优反应条件为: 底物 $n(\mathbf{1 a}): n(\mathbf{2 a})=$ $1.5: 1$, 配体 $\mathrm{PPh}_{3}$ 用量为 $20 \mathrm{~mol} \%, \mathrm{Pd}(\mathrm{OAc})_{2}$ 用量为 5 $\mathrm{mol} \%$, 反应溶剂为 $\mathrm{CH}_{3} \mathrm{CN}$, 反应温度为 $85{ }^{\circ} \mathrm{C}$, 反应时 间为 $24 \mathrm{~h}$.

\section{2 反应底物拓展}

在最佳反应条件下，对烯丙基碳酸酯与酰腙反应的 底物适应性进行了探索. 首先, 探索了不同取代基的烯 丙基碳酸酯和酰腙的烯丙基化反应，反应结果列于表 2 . 实验结果表明, 烯丙基碳酸酯衍生物的苯环上带有吸电 
子基或者供电子基时，反应都可以很好地发生(Entries 1 5). 当苯环的取代基为 4-甲基时, $3 \mathbf{b a}$ 的产物为 70\%; 当苯环的取代基为 4-F 时, 3ea 产率为 95\%, 这说明苯环 上带吸电子基的肉桂基碳酸酯具有更高的反应活性. 取 代基的位置对反应也有影响，例如苯环上取代基为 $4-\mathrm{OCH}_{3}$ 时, 产率为 $62 \%$, 而苯环上取代基为 $2-\mathrm{OCH}_{3}$ 时, 产率为 $81 \%$. 以上结果表明碳酸酯的位阻效应对反应结 果影响不大, 电子效应对产率的影响很大. 肉桂基碳酸 酯化合物的 $\mathrm{R}^{2}$ 取代基为长链烷基时，反应也可以发生， 产率可达 90\%.

表 2 肉桂基碳酸酯衍生物的底物范围 ${ }^{a}$

Table 2 Scope of cinnamyl carbonates

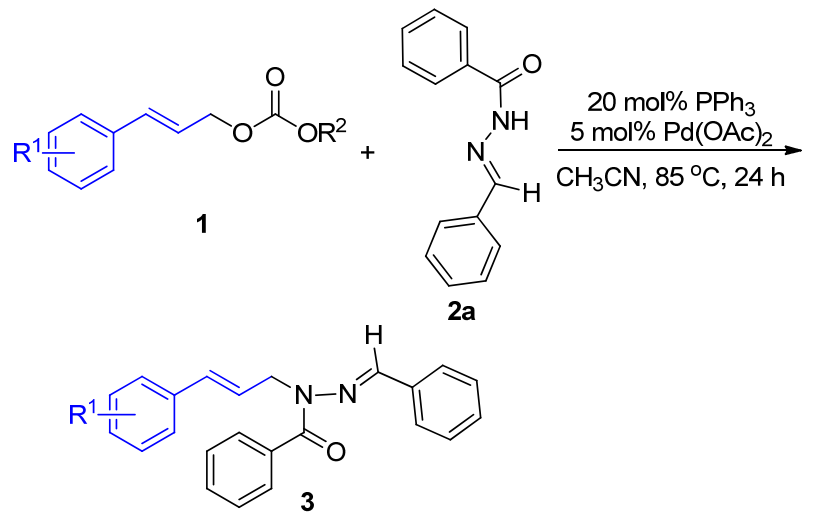

\begin{tabular}{|c|c|c|c|c|}
\hline Entry & $\mathrm{R}^{1}$ & $\mathrm{R}^{2}$ & Product & Yeild $^{b} / \%$ \\
\hline 1 & $4-\mathrm{CH}_{3}$ & $\mathrm{Me}$ & $3 \mathrm{ba}$ & 70 \\
\hline 2 & $4-\mathrm{OCH}_{3}$ & $\mathrm{Me}$ & $3 \mathrm{ca}$ & 62 \\
\hline 3 & $2-\mathrm{OCH}_{3}$ & $\mathrm{Me}$ & 3da & 80 \\
\hline 4 & $4-\mathrm{F}$ & $\mathrm{Me}$ & 3ea & 95 \\
\hline 5 & $\mathrm{H}$ & $\mathrm{CH}_{2} \mathrm{CH}_{2} \mathrm{CH}_{3}$ & $3 \mathbf{a a}$ & 90 \\
\hline
\end{tabular}

${ }^{a}$ Reaction condition: 1 (0.15 mmol), 2 a $(0.1 \mathrm{mmol}), \mathrm{PPh}_{3}(20 \mathrm{~mol} \%)$, $\mathrm{Pd}(\mathrm{OAc})_{2}(5 \mathrm{~mol} \%), \mathrm{CH}_{3} \mathrm{CN}, 85{ }^{\circ} \mathrm{C}, 24 \mathrm{~h}^{\circ}{ }^{b}$ Isolated yield.

随后我们研究了不同的芳香醛衍生的酰腙化合物 对 $N$-烯丙基化反应的影响, 反应结果列于表 3 . 由表 3 可知, $\mathrm{Ar}$ 上取代基的位置对产率的影响很大. 当甲氧基 在苯环上不同位置时, 产率有很大的差别: $3 \mathrm{ae}$ 的分离 产率略高于 3ac, 远高于 3ad 的产率(Entries 2 4). 苯环 上带有吸电子和供电子取代基时, 都可以中等到高的产 率得到相应的产物. 苯环 2 位带有供电子取代基的酰腙 化合物的反应活性低于苯环 2 位带有吸电子取代基的酰 腙化合物的反应活性(Entries 2, 5, 7). 然而, 苯环 4 位带 供电子取代基的酰腙(Entry 4)的反应活性要高于 4 位带 有吸电子取代基的酰腙的反应活性(Entries 6,8,10). 将 苯环换为荎基时(Entries 11, 12), 带 1-菜基的酰腙的反 应活性低于带 2-菜基的酰腙的反应活性. 两种杂环的酰 腙化合物(Entries 13,14)也可以顺利发生反应, 但 $3 a n$ 的 产率要高于 $3 \mathrm{ao}$ 的产率.
表 3 酰腙衍生物的范围 ${ }^{a}$

Table 3 Scope of acyl hydrazone compounds

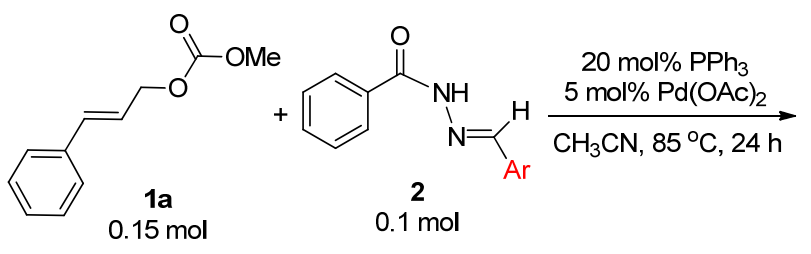<smiles>O=C(c1ccccc1)N(C/C=C/c1ccccc1)/N=C/[Bi]</smiles>

\begin{tabular}{|c|c|c|c|}
\hline Entry & $\mathrm{Ar}$ & Product & Yield $^{b} / \%$ \\
\hline 1 & $3-\mathrm{CH}_{3} \mathrm{C}_{6} \mathrm{H}_{4}$ & $3 a b$ & 71 \\
\hline 2 & $2-\mathrm{CH}_{3} \mathrm{OC}_{6} \mathrm{H}_{4}$ & $3 a c$ & 54 \\
\hline 3 & $3-\mathrm{CH}_{3} \mathrm{OC}_{6} \mathrm{H}_{4}$ & 3ad & 87 \\
\hline 4 & 4- $\mathrm{CH}_{3} \mathrm{OC}_{6} \mathrm{H}_{4}$ & $3 a e$ & 98 \\
\hline 5 & $2-\mathrm{FC}_{6} \mathrm{H}_{4}$ & $3 a f$ & 80 \\
\hline 6 & 4- $\mathrm{FC}_{6} \mathrm{H}_{4}$ & $3 a g$ & 79 \\
\hline 7 & $2-\mathrm{ClC}_{6} \mathrm{H}_{4}$ & 3ah & 62 \\
\hline 8 & $4-\mathrm{ClC}_{6} \mathrm{H}_{4}$ & 3ai & 69 \\
\hline 9 & $4-{ }^{t} \mathrm{BuC}_{6} \mathrm{H}_{4}$ & 3aj & 75 \\
\hline 10 & $4-\mathrm{NO}_{2} \mathrm{C}_{6} \mathrm{H}_{4}$ & 3ak & 86 \\
\hline 11 & 1-Naphthyl & 3al & 45 \\
\hline 12 & 2-Naphthyl & 3am & 88 \\
\hline 13 & 2-Furyl & 3 an & 94 \\
\hline 14 & 2-Thienyl & 3 ao & 70 \\
\hline
\end{tabular}

a Reaction condition: 1a $(0.15 \mathrm{mmol}), 2(0.1 \mathrm{mmol}), \mathrm{PPh}_{3}(20 \mathrm{~mol} \%)$, $\mathrm{Pd}(\mathrm{OAc})_{2}(5 \mathrm{~mol} \%), \mathrm{CH}_{3} \mathrm{CN}, 85{ }^{\circ} \mathrm{C}, 24 \mathrm{~h} .{ }^{b}$ Isolated yield.

\section{3 反应机理}

经查阅文献,我们推测了该反应的机理(Scheme 1): 首先,钯催化剂与配体形成钯 [0]络合物; 然后钯 [0]络合 物与肉桂基碳酸酯经过氧化加成, 形成了烯丙基钯中间 体; 接着, 酰腙上的 $\mathrm{N}$ 亲核进攻烯丙基钯中间体的 3 位, 形成了目标烯丙基胺, 同时完成催化剂钯[0]络合物的 再生.

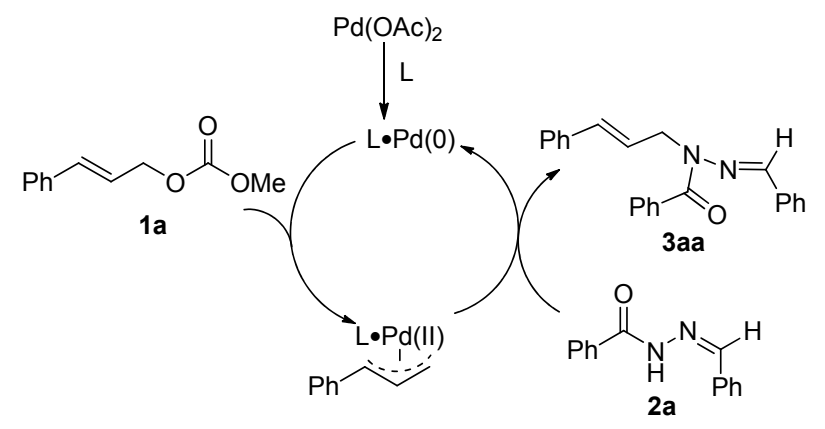

图式 1 可能的反应机理

Scheme 1 Possible mechanism 


\section{2 结论}

发展了一种 $\mathrm{Pd}(\mathrm{OAc})_{2} / \mathrm{PPh}_{3}$ 体系催化的肉桂基碳酸 酯和酰腙化合物的烯丙基胺化反应合成线型烯丙基胺 类化合物的方法. 该方法具有无需加碱、条件温和、操 作简单、区域选择性高、底物范围广等优点.

\section{3 实验部分}

\section{1 仪器与试剂}

核磁共振 ${ }^{1} \mathrm{H}$ NMR, ${ }^{13} \mathrm{C}$ NMR 用 Bruker AVANCE III $(400 \mathrm{MHz})$ 型超导核磁共振仪测定, TMS 为内标, $\mathrm{CDCl}_{3}$ 和氛代二甲基亚砜(DMSO- $d_{6}$ ) 为溶剂; 薄层色谱(TLC) 使用青岛海洋化工厂生产的 $\mathrm{GF}_{254}$ 薄层色谱硅胶, 柱色 谱使用青岛海洋化工厂生产的 200 300 目柱层析硅胶.

除非特别指出, 本实验所用的药品、试剂均为买来 后直接使用. 其中, 无水乙腈、二氯甲烷加入氢化钙回 流 $2 \mathrm{~h}$, 然后蒸馏制得; 无水吡啶是先与颗粒状的氢氧 化钾回流, 然后隔绝空气蒸馏; 无水四氢呋喃(THF)是 先加钠丝与二苯甲酮(指示剂)加热回流至蓝色, 然后蒸 馏制得.

\section{2 实验方法}

取底物 $1 \mathbf{a}(0.15 \mathrm{mmol}, 28.8 \mathrm{mg})$, 底物 $2 \mathbf{a}(0.1 \mathrm{mmol}$, $22.4 \mathrm{mg}$ ), $\mathrm{PPh}_{3}$ (20 mol\%, $\left.5.2 \mathrm{mg}\right), \mathrm{Pd}(\mathrm{OAc})_{2}$ (5 mol\%, 1.1 $\mathrm{mg}$ )于 $10 \mathrm{~mL}$ Schlenk 反应管中, 在氩气的保护下加入 2 $\mathrm{mL} \mathrm{CH}{ }_{3} \mathrm{CN}, 85{ }^{\circ} \mathrm{C}$ 下反应 $24 \mathrm{~h}$. TLC 方法检测, 待反应 完全后直接柱色谱分离提纯 [展开剂: $V$ (乙酸乙酯) : $V$ (石油醚 $)=1: 5$ ] 即可得到 $N^{\prime}$-芐基- $N$-肉桂基苯甲酰肼 (3aa) $33.8 \mathrm{mg}$, 产率为 99\%. 白色固体, m.p. 110 $113{ }^{\circ} \mathrm{C} ;{ }^{1} \mathrm{H}$ NMR (400 MHz, $\mathrm{CDCl}_{3}$ ) $\delta: 7.84$ (s, 1H), 7.80 (d, $J=4.0 \mathrm{~Hz}, 2 \mathrm{H}), 7.47$ (br, 5H), $7.41 \sim 7.20(\mathrm{~m}, 8 \mathrm{H})$, $6.58(\mathrm{~d}, J=16.0 \mathrm{~Hz}, 1 \mathrm{H}), 6.27$ (d, $J=16.0 \mathrm{~Hz}, 1 \mathrm{H}), 5.01$ $(\mathrm{s}, 2 \mathrm{H}) ;{ }^{13} \mathrm{C} \mathrm{NMR}\left(101 \mathrm{MHz}, \mathrm{CDCl}_{3}\right) \delta: 170.96,139.95$, $136.20,135.04,134.59,132.31,130.33,129.99,129.58$, $128.69,128.59,127.87,127.42,127.21,126.41,122.04$, 43.52; HRMS (ESI) calcd for $\mathrm{C}_{23} \mathrm{H}_{21} \mathrm{~N}_{2} \mathrm{O}[\mathrm{M}+\mathrm{H}]^{+}$ 341.1648 , found 341.1652 .

$N^{\prime}$-亚芳基- $N$ - $[(E)$-3-(对甲苯基)烯丙基]苯甲酰肼 (3ba): 白色固体, 产率 70\%. m.p. 58 60 ${ }^{\circ} \mathrm{C} ;{ }^{1} \mathrm{H} \mathrm{NMR}$ $\left(400 \mathrm{MHz}, \mathrm{CDCl}_{3}\right) \delta: 7.84(\mathrm{~s}, 1 \mathrm{H}), 7.80(\mathrm{~d}, J=8.0 \mathrm{~Hz}$, 2H), $7.58 \sim 7.20(\mathrm{~m}, 10 \mathrm{H}), 7.11(\mathrm{~d}, J=8.0 \mathrm{~Hz}, 2 \mathrm{H}), 6.55$ (d, $J=16.0 \mathrm{~Hz}, 1 \mathrm{H}), 6.21(\mathrm{~d}, J=16.0 \mathrm{~Hz}, 1 \mathrm{H}), 5.00$ (s, 2H), $2.32(\mathrm{~s}, 3 \mathrm{H}) ;{ }^{13} \mathrm{C}$ NMR (101 MHz, $\left.\mathrm{CDCl}_{3}\right) \delta: 170.96$, $139.96,137.75,135.13,134.66,133.42,132.28,130.35$, $129.98,129.63,129.28,128.67,127.40,127.20,126.31$, 120.94, 43.58, 21.19; HRMS (ESI) cald for $\mathrm{C}_{24} \mathrm{H}_{23} \mathrm{~N}_{2} \mathrm{O}$
$[\mathrm{M}+\mathrm{H}]^{+}$355.1805, found 355.1806.

$N^{\prime}$-亚苄基- $N$ - $[(E)$-3-(4-甲氧基苯基)烯丙基]苯甲酰 肼(3ca): 白色固体，产率 62\%. m.p. 156 $158{ }^{\circ} \mathrm{C} ;{ }^{1} \mathrm{H}$ NMR (400 MHz, $\left.\mathrm{CDCl}_{3}\right) \delta: 7.84(\mathrm{~s}, 1 \mathrm{H}), 7.80$ (d, $J=4.0$ $\mathrm{Hz}, 2 \mathrm{H}), 7.47$ (br, 5H), 7.31 (br, 5H), 6.84 (d, J=8.0 Hz, $2 \mathrm{H}), 6.53(\mathrm{~d}, J=16.0 \mathrm{~Hz}, 1 \mathrm{H}), 6.12(\mathrm{~d}, J=16.0 \mathrm{~Hz}, 1 \mathrm{H})$, 4.99 (s, 2H), 3.79 (s, 3H); $\left.{ }^{13} \mathrm{C} \mathrm{NMR} \mathrm{(101} \mathrm{MHz,} \mathrm{CDCl}_{3}\right) \delta$ : $170.96,159.38,139.93,135.16,134.70,131.92,130.34$, $129.98,129.63,129.02,128.68,127.61,127.40,127.20$, 119.71, 113.98, 55.30, 43.61; HRMS (ESI) calcd for $\mathrm{C}_{24} \mathrm{H}_{23} \mathrm{~N}_{2} \mathrm{O}_{2}[\mathrm{M}+\mathrm{H}]^{+}$371.1754, found 371.1755.

$N^{\prime}$-亚芳基- $N$ - $[(E)$-3-(2-甲氧基苯基)烯丙基]苯甲酰 肼(3da): 白色固体，产率 $80 \%$. m.p. $79 \sim 81{ }^{\circ} \mathrm{C} ;{ }^{1} \mathrm{H}$ NMR (400 MHz, $\left.\mathrm{CDCl}_{3}\right) \delta: 7.91(\mathrm{~s}, 1 \mathrm{H}), 7.79$ (d, $J=8.0$ $\mathrm{Hz}, 2 \mathrm{H}), 7.55 \sim 7.36(\mathrm{~m}, 5 \mathrm{H}), 7.35 \sim 7.16(\mathrm{~m}, 4 \mathrm{H}), 7.02 \sim$ $6.80(\mathrm{~m}, 3 \mathrm{H}), 6.25(\mathrm{~d}, J=16.0 \mathrm{~Hz}, 1 \mathrm{H}), 5.03(\mathrm{~s}, 2 \mathrm{H}), 3.81$ $(\mathrm{s}, 3 \mathrm{H}) ;{ }^{13} \mathrm{C}$ NMR $\left(101 \mathrm{MHz}, \mathrm{CDCl}_{3}\right) \delta: 170.98,156.65$, 140.12 , 135.30, 134.79, 130.25, 129.93, 129.54, 128.89, $128.62,128.00,127.37,127.19,127.00,125.33,122.89$, 120.62, 110.83, 55.43, 44.19; HRMS (ESI) calcd for $\mathrm{C}_{24} \mathrm{H}_{23} \mathrm{~N}_{2} \mathrm{O}_{2}[\mathrm{M}+\mathrm{H}]^{+}$371.1754, found 371.1760.

$N^{\prime}$ - 亚芳基- $N$ - $[(E)$-3-(4-氟苯基)烯丙基]苯甲酰肼 (3ea): 浅黄色固体, 产率 95\%. m.p. 97 99 ${ }^{\circ} \mathrm{C} ;{ }^{1} \mathrm{H} \mathrm{NMR}$ $\left(400 \mathrm{MHz}, \mathrm{CDCl}_{3}\right) \delta: 7.81$ (br, 3H), 7.47 (s, 5H), 7.32 (s, $5 \mathrm{H}), 6.99$ (s, 2H), $6.53(\mathrm{~d}, J=16.0 \mathrm{~Hz}, 1 \mathrm{H}), 6.18$ (d, $J=$ $16.0 \mathrm{~Hz}, 1 \mathrm{H}), 4.99$ (s, 2H); ${ }^{13} \mathrm{C} \mathrm{NMR}\left(101 \mathrm{MHz}, \mathrm{CDCl}_{3}\right) \delta$ : $170.95,162.42$ (d, $J=247.5 \mathrm{~Hz}), 139.90,135.00,134.59$, $132.36(\mathrm{~d}, J=4.0 \mathrm{~Hz}), 131.16,130.44,130.00,129.71$, $128.71,127.95$ (d, $J=8.1 \mathrm{~Hz}), 127.43,127.20,121.71$ (d, $J=2.0 \mathrm{~Hz}$ ), 115.51 (d, $J=21.2 \mathrm{~Hz}$ ), 43.39; HRMS (ESI) calcd for $\mathrm{C}_{23} \mathrm{H}_{20} \mathrm{FN}_{2} \mathrm{O}[\mathrm{M}+\mathrm{H}]^{+} 359.1554$, found 359.1560 .

$N$-肉桂基- $N^{\prime}$-(3-甲基苯亚甲基)苯甲酰肼(3ab): 浅 黄色固体，产率 71\%. m.p. 92 94 ${ }^{\circ} \mathrm{C} ;{ }^{1} \mathrm{H}$ NMR (400 $\left.\mathrm{MHz}, \mathrm{CDCl}_{3}\right) \delta: 7.81(\mathrm{~s}, 3 \mathrm{H}), 7.47(\mathrm{br}, 3 \mathrm{H}), 7.41 \sim 7.16$ $(\mathrm{m}, 8 \mathrm{H}), 7.14(\mathrm{~s}, 1 \mathrm{H}), 6.56(\mathrm{~d}, J=16.0 \mathrm{~Hz}, 1 \mathrm{H}), 6.27$ (d, $J=12.0 \mathrm{~Hz}, 1 \mathrm{H}), 5.01$ (s, 2H), $2.30(\mathrm{~s}, 3 \mathrm{H}) ;{ }^{13} \mathrm{C} \mathrm{NMR}(101$ $\left.\mathrm{MHz}, \mathrm{CDCl}_{3}\right) \delta: 170.96,140.14,138.32,136.21,135.07$, $134.57,132.27,130.47,130.39,130.08,128.58,128.04$, 127.84, 127.36, 126.41, 124.26, 122.05, 43.65, 20.92; HRMS (ESI) calcd for $\mathrm{C}_{24} \mathrm{H}_{23} \mathrm{~N}_{2} \mathrm{O}[\mathrm{M}+\mathrm{H}]^{+} 355.1805$, found 355.1809 .

$N$-肉桂基- $N^{\prime}$-(2-甲氧基苯亚甲基)苯甲酰肼(3ac): 白色固体, 产率 54\%. m.p. 113 $115{ }^{\circ} \mathrm{C} ;{ }^{1} \mathrm{H}$ NMR (400 $\left.\mathrm{MHz}, \mathrm{CDCl}_{3}\right) \delta: 8.30(\mathrm{~s}, 1 \mathrm{H}), 7.79(\mathrm{~d}, J=4.0 \mathrm{~Hz}, 2 \mathrm{H})$, $7.54(\mathrm{~d}, J=4.0 \mathrm{~Hz}, 1 \mathrm{H}), 7.50 \sim 7.15(\mathrm{~m}, 9 \mathrm{H}), 6.86(\mathrm{~d}, J=$ 
$4.0 \mathrm{~Hz}, 2 \mathrm{H}), 6.63(\mathrm{~d}, J=16.0 \mathrm{~Hz}, 1 \mathrm{H}), 6.26(\mathrm{~d}, J=16.0$ $\mathrm{Hz}, 1 \mathrm{H}), 5.02(\mathrm{~d}, J=4.0 \mathrm{~Hz}, 2 \mathrm{H}), 3.82(\mathrm{~s}, 3 \mathrm{H}) ;{ }^{13} \mathrm{C} \mathrm{NMR}$ $\left(101 \mathrm{MHz}, \mathrm{CDCl}_{3}\right) \delta: 170.95,158.00,136.45,136.31$, $135.33,132.86,130.83,130.23,129.97,128.54,127.74$, 127.36, 126.42, 125.95, 123.11, 122.18, 120.85, 111.00, 55.59, 43.64; HRMS (ESI) calcd for $\mathrm{C}_{24} \mathrm{H}_{23} \mathrm{~N}_{2} \mathrm{O}_{2}[\mathrm{M}+\mathrm{H}]^{+}$ 371.1754 , found 371.1758 .

$N$-肉桂基- $N^{\prime}$-(3-甲氧基苯亚甲基)苯甲酰肼(3ad): 白色固体, 产率 $87 \%$. m.p. $97 \sim 99{ }^{\circ} \mathrm{C} ;{ }^{1} \mathrm{H}$ NMR (400 $\left.\mathrm{MHz}, \mathrm{CDCl}_{3}\right) \delta: 7.79$ (br, $\left.3 \mathrm{H}\right), 7.45(\mathrm{~d}, J=8.0 \mathrm{~Hz}, 3 \mathrm{H})$, $7.40 \sim 7.13(\mathrm{~m}, 6 \mathrm{H}), 7.03(\mathrm{br}, 2 \mathrm{H}), 6.87(\mathrm{~d}, J=4.0 \mathrm{~Hz}$, $1 \mathrm{H}), 6.58(\mathrm{~d}, J=16.0 \mathrm{~Hz}, 1 \mathrm{H}), 6.26(\mathrm{~d}, J=16.0 \mathrm{~Hz}, 1 \mathrm{H})$, $5.00(\mathrm{~d}, J=4.0 \mathrm{~Hz}, 2 \mathrm{H}), 3.71$ (s, 3H); ${ }^{13} \mathrm{C}$ NMR $(101 \mathrm{MHz}$, $\left.\mathrm{CDCl}_{3}\right) \delta: 170.96,159.80,139.46,136.20,136.11,135.21$, $132.46,130.30,129.97,129.66,128.60,127.88,127.32$, 126.42, 121.96, 120.56, 116.49, 110.44, 55.11, 43.49; HRMS (ESI) calcd for $\mathrm{C}_{24} \mathrm{H}_{23} \mathrm{~N}_{2} \mathrm{O}_{2}[\mathrm{M}+\mathrm{H}]^{+} 371.1754$, found 371.1760 .

$N$-肉桂基- $N^{\prime}$-(4-甲氧基苯亚甲基)苯甲酰肼(3ae): 白色固体, 产率 $98 \%$. m.p. $114 \sim 115{ }^{\circ} \mathrm{C} ;{ }^{1} \mathrm{H}$ NMR (400 $\left.\mathrm{MHz}, \mathrm{CDCl}_{3}\right) \delta: 7.80(\mathrm{~s}, 3 \mathrm{H}), 7.60 \sim 7.14(\mathrm{~m}, 10 \mathrm{H}), 6.84$ (d, $J=8.0 \mathrm{~Hz}, 2 \mathrm{H}), 6.57$ (d, $J=16.0 \mathrm{~Hz}, 1 \mathrm{H}), 6.27$ (d, $J=$ $16.0 \mathrm{~Hz}, 1 \mathrm{H}), 4.99$ (s, 2H), 3.79 (s, 3H); ${ }^{13} \mathrm{C}$ NMR (101 $\left.\mathrm{MHz}, \mathrm{CDCl}_{3}\right) \delta: 170.79,160.86,139.80,136.29,135.32$, $132.27,130.25,129.95,128.68,128.58,127.82,127.47$, 127.37, 126.41, 122.34, 114.14, 55.33, 43.51; HRMS (ESI) calcd for $\mathrm{C}_{24} \mathrm{H}_{23} \mathrm{~N}_{2} \mathrm{O}_{2}[\mathrm{M}+\mathrm{H}]^{+} 371.1754$, found 371.1760 .

$N$-肉桂基- $N^{\prime}-(2$-氟苯亚甲基)苯甲酰肼(3af): 浅黄 色固体, 产率 $80 \%$. m.p. $81 \sim 82{ }^{\circ} \mathrm{C} ;{ }^{1} \mathrm{H}$ NMR $(400 \mathrm{MHz}$, $\left.\mathrm{CDCl}_{3}\right) \delta: 8.11(\mathrm{~s}, 1 \mathrm{H}), 7.78(\mathrm{~d}, J=8.0 \mathrm{~Hz}, 2 \mathrm{H}), 7.65 \sim$ $7.16(\mathrm{~m}, 10 \mathrm{H}), 7.04(\mathrm{~d}, J=8.0 \mathrm{~Hz}, 2 \mathrm{H}), 6.62(\mathrm{~d}, J=16.0$ $\mathrm{Hz}, 1 \mathrm{H}), 6.24(\mathrm{~d}, J=16.0 \mathrm{~Hz}, 1 \mathrm{H}), 5.02(\mathrm{~s}, 2 \mathrm{H}) ;{ }^{13} \mathrm{C} \mathrm{NMR}$ $\left(101 \mathrm{MHz}, \mathrm{CDCl}_{3}\right) \delta: 171.04,161.50(\mathrm{~d}, J=252.5 \mathrm{~Hz})$, 136.23, 135.03, 133.14, 133.07, 131.04 (d, $J=8.1 \mathrm{~Hz})$, $130.44,129.93,128.59,127.90,127.44,126.47,126.35(\mathrm{~d}$, $J=3.0 \mathrm{~Hz}), 124.40(\mathrm{~d}, J=4.0 \mathrm{~Hz}), 122.46(\mathrm{~d}, J=10.1 \mathrm{~Hz})$, 121.69, 115.76 (d, $J=21.2 \mathrm{~Hz}), 43.67$; HRMS (ESI) calcd for $\mathrm{C}_{23} \mathrm{H}_{20} \mathrm{FN}_{2} \mathrm{O}[\mathrm{M}+\mathrm{H}]^{+}$359.1554, found 359.1558.

$N$-肉桂基- $N$ '-(4-氟苯亚甲基)苯甲酰肼(3ag): 黄色 固体, 产率 79\%. m.p. $92 \sim 93{ }^{\circ} \mathrm{C} ;{ }^{1} \mathrm{H}$ NMR $(400 \mathrm{MHz}$, $\left.\mathrm{CDCl}_{3}\right) \delta: 7.80(\mathrm{br}, 3 \mathrm{H}), 7.60 \sim 7.15(\mathrm{~m}, 10 \mathrm{H}), 7.00(\mathrm{br}$, 2H), $6.57(\mathrm{~d}, J=16.0 \mathrm{~Hz}, 1 \mathrm{H}), 6.26(\mathrm{~d}, J=16.0 \mathrm{~Hz}, 1 \mathrm{H})$, $5.00(\mathrm{~s}, 2 \mathrm{H}) ;{ }^{13} \mathrm{C}$ NMR $\left(101 \mathrm{MHz}, \mathrm{CDCl}_{3}\right) \delta: 170.93$, $163.51(\mathrm{~d}, J=251.5 \mathrm{~Hz}), 138.67,136.14,135.06,132.39$, 130.89 (d, $J=3.0 \mathrm{~Hz}), 130.42,129.88,128.95$ (d, $J=8.1$
Hz), 128.62, 127.92, 127.45, 126.40, 121.98, 115.81 (d, $J=22.2 \mathrm{~Hz}$ ), 43.56; HRMS (ESI) calcd for $\mathrm{C}_{23} \mathrm{H}_{20} \mathrm{FN}_{2} \mathrm{O}$ $[\mathrm{M}+\mathrm{H}]^{+}$359.1554, found 359.1556.

$N$-肉桂基- $N^{\prime}$-(2-氯苯亚甲基)苯甲酰肼(3ah): 浅黄 色固体, 产率 62\%. m.p. $74 \sim 75{ }^{\circ} \mathrm{C} ;{ }^{1} \mathrm{H}$ NMR $(400 \mathrm{MHz}$, $\left.\mathrm{CDCl}_{3}\right) \delta: 8.33(\mathrm{~s}, 1 \mathrm{H}), 7.77(\mathrm{~d}, J=4.0 \mathrm{~Hz}, 2 \mathrm{H}), 7.59(\mathrm{~d}$, $J=4.0 \mathrm{~Hz}, 1 \mathrm{H}), 7.54 \sim 7.05(\mathrm{~m}, 11 \mathrm{H}), 6.69(\mathrm{~d}, J=16.0 \mathrm{~Hz}$, 1H), $6.23(\mathrm{~d}, J=16.0 \mathrm{~Hz}, 1 \mathrm{H}), 5.04(\mathrm{~s}, 2 \mathrm{H}) ;{ }^{13} \mathrm{C}$ NMR $(101$ $\left.\mathrm{MHz}, \mathrm{CDCl}_{3}\right) \delta: 171.08,136.93,136.20,135.01,134.38$, $133.79,131.95,130.47,129.91,129.76,128.60,127.93$, $127.47,127.05,126.77,126.50,121.74,44.11$; HRMS (ESI) calcd for $\mathrm{C}_{23} \mathrm{H}_{20} \mathrm{ClN}_{2} \mathrm{O}[\mathrm{M}+\mathrm{H}]^{+} 375.1259$, found 375.1262 .

$N$-肉桂基- $N^{\prime}$-(4-氯苯亚甲基)苯甲酰肼(3ai): 黄色固 体, 产率 69\%. m.p. $78 \sim 80{ }^{\circ} \mathrm{C} ;{ }^{1} \mathrm{H}$ NMR $(400 \mathrm{MHz}$, $\left.\mathrm{CDCl}_{3}\right) \delta: 7.78(\mathrm{~s}, 3 \mathrm{H}), 7.60 \sim 7.13(\mathrm{~m}, 12 \mathrm{H}), 6.56(\mathrm{~d}, J=$ $16.0 \mathrm{~Hz}, 1 \mathrm{H}), 6.25(\mathrm{~d}, J=16.0 \mathrm{~Hz}, 1 \mathrm{H}), 5.00(\mathrm{~s}, 2 \mathrm{H}) ;{ }^{13} \mathrm{C}$ NMR $\left(101 \mathrm{MHz}, \mathrm{CDCl}_{3}\right) \delta: 170.93,138.50,136.09$, $135.42,134.94,133.16,132.45,130.49,129.90,128.94$, 128.61, 128.31, 127.94, 127.45, 126.40, 121.85, 43.60; HRMS (ESI) calcd for $\mathrm{C}_{23} \mathrm{H}_{20} \mathrm{ClN}_{2} \mathrm{O}[\mathrm{M}+\mathrm{H}]^{+} 375.1259$, found 375.1263 .

$N$-肉桂基- $N^{\prime}$-(4-叔丁基苯亚甲基)苯甲酰肼(3aj): 黄色粘稠液体, 产率 75\%. ${ }^{1} \mathrm{H}$ NMR $\left(400 \mathrm{MHz}, \mathrm{CDCl}_{3}\right) \delta$ : $7.81(\mathrm{~d}, J=4.0 \mathrm{~Hz}, 3 \mathrm{H}), 7.58 \sim 7.12(\mathrm{~m}, 12 \mathrm{H}), 6.55(\mathrm{~d}, J=$ $16.0 \mathrm{~Hz}, 1 \mathrm{H}), 6.26(\mathrm{~d}, J=12.0 \mathrm{~Hz}, 1 \mathrm{H}), 4.99$ (s, 2H), 1.28 (s, 9H); ${ }^{13} \mathrm{C}$ NMR $\left(101 \mathrm{MHz}, \mathrm{CDCl}_{3}\right) \delta: 171.00,153.08$, $140.09,136.15,135.08,132.21,131.85,130.34,129.96$, $128.55,127.81,127.38,126.99,126.37,125.65,121.95$, 43.48, 34.87, 31.22; HRMS (ESI) calcd for $\mathrm{C}_{27} \mathrm{H}_{29} \mathrm{~N}_{2} \mathrm{O}$ $[\mathrm{M}+\mathrm{H}]^{+}$397.2274, found 397.2277.

$N$-肉桂基- $N^{\prime}$-(4-硝基苯亚甲基)苯甲酰肼(3ak): 黄 色固体, 产率 $86 \%$. m.p. $142 \sim 145{ }^{\circ} \mathrm{C} ;{ }^{1} \mathrm{H}$ NMR (400 $\left.\mathrm{MHz}, \mathrm{CDCl}_{3}\right) \delta: 8.18(\mathrm{br}, 2 \mathrm{H}), 8.00 \sim 7.18(\mathrm{~m}, 13 \mathrm{H}), 6.58$ (d, $J=16.0 \mathrm{~Hz}, 1 \mathrm{H}), 6.26(\mathrm{~d}, J=8.0 \mathrm{~Hz}, 1 \mathrm{H}), 5.03(\mathrm{~s}, 2 \mathrm{H})$; ${ }^{13} \mathrm{C}$ NMR $\left(101 \mathrm{MHz}, \mathrm{CDCl}_{3}\right) \delta: 171.14,147.95,140.65$, $136.78,135.88,134.51,132.79,130.84,129.87,128.68$, $128.12,127.61,126.42,124.04,121.28,43.85$; HRMS (ESI) calcd for $\mathrm{C}_{23} \mathrm{H}_{20} \mathrm{~N}_{3} \mathrm{O}_{3}[\mathrm{M}+\mathrm{H}]^{+} 386.1499$, found 386.1501.

$N$-肉桂基- $N^{\prime}$-(1-萗基亚甲基)苯甲酰肼(3al): 浅黄色 固体, 产率 45\%. m.p. $108 \sim 110{ }^{\circ} \mathrm{C} ;{ }^{1} \mathrm{H}$ NMR $(400 \mathrm{MHz}$, $\left.\mathrm{CDCl}_{3}\right) \delta: 8.45(\mathrm{~s}, 1 \mathrm{H}), 8.34(\mathrm{~d}, J=8.0 \mathrm{~Hz}, 1 \mathrm{H}), 7.80(\mathrm{~s}$, $4 \mathrm{H}), 7.68 \sim 7.14(\mathrm{~m}, 12 \mathrm{H}), 6.70(\mathrm{~d}, J=16.0 \mathrm{~Hz}, 1 \mathrm{H}), 6.35$ (d, $J=12.0 \mathrm{~Hz}, 1 \mathrm{H}), 5.13$ (s, 2H); ${ }^{13} \mathrm{C}$ NMR $(101 \mathrm{MHz}$, 
$\left.\mathrm{CDCl}_{3}\right) \delta: 171.61,140.79,136.15,135.64,133.80,132.69$, $130.48,130.25,130.09,129.75,129.28,128.65,128.62$, $128.58,127.92,127.73,126.94,126.42,126.02,125.20$, 124.56, 122.29, 43.36; HRMS (ESI) calcd for $\mathrm{C}_{27} \mathrm{H}_{23} \mathrm{~N}_{2} \mathrm{O}$ $[\mathrm{M}+\mathrm{H}]^{+}$391.1805, found 391.1807.

$N$-肉桂基- $N^{\prime}$-(2-萗基亚甲基)苯甲酰肼(3am): 白色 固体, 产率 88\%. m.p. 77 78 ${ }^{\circ} \mathrm{C} ;{ }^{1} \mathrm{H}$ NMR (400 MHz, $\left.\mathrm{CDCl}_{3}\right) \delta: 8.00(\mathrm{~s}, 1 \mathrm{H}), 7.91 \sim 7.15(\mathrm{~m}, 17 \mathrm{H}), 6.62(\mathrm{~d}, J=$ $16.0 \mathrm{~Hz}, 1 \mathrm{H}), 6.31(\mathrm{~d}, J=16.0 \mathrm{~Hz}, 1 \mathrm{H}), 5.06(\mathrm{~s}, 2 \mathrm{H}) ;{ }^{13} \mathrm{C}$ NMR (101 $\left.\mathrm{MHz}, \mathrm{CDCl}_{3}\right) \delta: 170.96,140.07,136.18$, $135.08,133.96,133.07,132.39$, 132.35, 130.45, 130.03, $129.08,128.60$, 128.19, 127.88, 127.84, 127.45, 126.87, 126.55, 126.42, 122.71, 122.07, 43.61; HRMS (ESI) calcd for $\mathrm{C}_{27} \mathrm{H}_{23} \mathrm{~N}_{2} \mathrm{O}[\mathrm{M}+\mathrm{H}]^{+}$391.1805, found 391.1808.

$N$-肉桂基- $N^{\prime}$-(2-呋喃基亚甲基)苯甲酰肼(3an)：黄 色固体, 产率 94\%. m.p. 106 $107{ }^{\circ} \mathrm{C} ;{ }^{1} \mathrm{H}$ NMR (400 $\left.\mathrm{MHz} \mathrm{CDCl}_{3}\right) \delta: 7.85(\mathrm{br}, 2 \mathrm{H}), 7.74(\mathrm{~s}, 1 \mathrm{H}), 7.57 \sim 7.19$ (m, 9H), 6.55 (br, 2H), $6.42(\mathrm{~s}, 1 \mathrm{H}), 6.24$ (d, $J=16.0 \mathrm{~Hz}$, $1 \mathrm{H}), 4.97$ (s, 2H); ${ }^{13} \mathrm{C}$ NMR (101 MHz, $\left.\mathrm{CDCl}_{3}\right) \delta: 170.47$, $150.33,143.90,136.14,134.50,132.38,130.59,130.32$, $128.60,127.90,127.43,126.43,121.74,111.78,111.20$, 43.72; HRMS (ESI) calcd for $\mathrm{C}_{21} \mathrm{H}_{19} \mathrm{~N}_{2} \mathrm{O}_{2}[\mathrm{M}+\mathrm{H}]^{+}$ 331.1441, found 331.1443.

$N$-肉桂基- $N^{\prime}$-(2-噻吩基亚甲基)苯甲酰肼(3ao)：黄 色固体, 产率 $70 \%$. m.p. $100 \sim 102{ }^{\circ} \mathrm{C} ;{ }^{1} \mathrm{H}$ NMR (400 $\left.\mathrm{MHz}, \mathrm{CDCl}_{3}\right) \delta: 8.00(\mathrm{~s}, 1 \mathrm{H}), 7.82(\mathrm{~d}, J=4.0 \mathrm{~Hz}, 2 \mathrm{H})$, $7.61 \sim 7.09(\mathrm{~m}, 9 \mathrm{H}), 6.99(\mathrm{~s}, 1 \mathrm{H}), 6.57(\mathrm{~d}, J=16.0 \mathrm{~Hz}$, $1 \mathrm{H}), 6.25(\mathrm{~d}, J=12.0 \mathrm{~Hz}, 1 \mathrm{H}), 4.98(\mathrm{~s}, 2 \mathrm{H}) ;{ }^{13} \mathrm{C}$ NMR $(101$ $\left.\mathrm{MHz}, \mathrm{CDCl}_{3}\right) \delta: 170.40,140.28,136.14,134.54,134.43$, $132.38,130.48,130.29,129.07,128.61,127.91,127.75$, 127.41, 127.35, 126.43, 121.91, 43.82; HRMS (ESI) calcd for $\mathrm{C}_{21} \mathrm{H}_{19} \mathrm{~N}_{2} \mathrm{SO}[\mathrm{M}+\mathrm{H}]^{+}$347.1213, found 347.1216.

辅助材料(Supporting Information) 化合物 3aa $\sim 3 \mathbf{a o}$ 和 $3 \mathrm{ba} \sim 3 \mathrm{ea}$ 的核磁谱图. 这些材料可以免费从本刊网 站(http://sioc-journal. cn/)上下载.

\section{References}

[1] (a) Brown, E. G. Ring Nitrogen and Key Biomolecules, Springer, Boston, MA, 1998.

(b) Yu, X. Y.; Zhou, F.; Chen, J. R.; Xiao, W. J. Acta Chim. Sinica 2017, 75, 86 (in Chinese)

(余晓叶, 周帆, 陈加荣, 肖文精, 化学学报, 2017, 75, 86.)

(c) Li, W. F.; Ma, Q.; Zheng, Z. Z.; Zhang, Y. G. Acta Chim. Sinica 2017, 75, 225 (in Chinese).

(李宛飞, 马倩, 郑召召, 张跃钢, 化学学报, 2017, 75, 225.)

(d) Li, T. T.; Zhao, J. K.; Li, R.; Quan, Z. L.; Xu, J. Acta Chim. Sinica 2017, 75, 485 (in Chinese).

(李甜甜, 赵继宽, 李尧, 全贞兰, 徐洁, 化学学报, 2017, 75,
485.)

[2] (a) Trost, B. M.; Crawley, M. L. Chem. Rev. 2003, 103, 2921.

(b) Trost, B. M.; Pissot-Soldermann, C.; Chen, I.; Schroede, G. M. J. Am. Chem. Soc. 2004, 126, 4480

(c) Wang, D.; Yu, X.; Ge, B.; Miao, H.; Ding, Y. Chin. J. Org. Chem. 2015, 35, 676 (in Chinese).

(王大伟，余晓丽，葛冰洋，苗红艳，丁玉强，有机化学，2015, $35,676$.

(d) Hu, X.; Yang, B.; Yao, W.; Wang, D. Chin. J. Org. Chem. 2018, 38, 3296 (in Chinese).

(胡欣宇，杨伯斌，姚玮，王大伟，有机化学, 2018, 38, 3296.)

[3] (a) Monk, J. P.; Brogden, R. N. Drugs 1991, 42, 659.

(b) Birnbaum, J. E. J. Am. Acad. Dermatol. 1990, 23, 782.

(c) Xu, Z.; Wang, D. S.; Yu, X.; Yang Y.; Wang, D. Adv. Synth. Catal. 2017, 359, 3332.

[4] (a) Stuetz, A.; Petranyi, G. J. Med. Chem. 1984, 27, 1539.

(b) Rudisill, D. E.; Castonguay, L. A.; Me, J. K. Tetrahedron Lett. 1988, 29, 1509.

(c) Balfour, J. A.; Fauids, D. Drugs 1992, 43, 259

(d) Ge, C.; Sang, X.; Yao, W.; Zhang L.; Wang, D. Green Chem. 2018, 20, 1805.

[5] (a) Andersson, P. G.; Backvall, J. E. In Handbook of Organopalladium Chemistry for Organic Synthesis, Ed.: Negishi, E., WileyInterscience, New York, 2002, p. 1859.

(b) Davies, H. M. L.; Long, M. S. Angew. Chem., Int. Ed. 2005, 44, 3518 .

(c) Jiang, L.; Buchwald, S. L. In Metal-Catalyzed Cross-Coupling Reactions, 2nd ed., Wiley-VCH, Weinheim, 2004, Vol. 2, p. 699.

(d) Hartwig, J. F. In Handbook of Organopalladium Chemistry for Organic Synthesis, Ed.: Negishi, E., Wiley-Interscience, New York, 2002, p. 1051

[6] (a) Ranerand, K. D.; Ward, A. D. Aust. J. Chem. 1991, 44, 1749. (b) Cooper, M. A.; Lucas, M. A.; Taylor, J. M.; Ward, A. D.; Williamson, N. M. Synthesis 2001, 621.

(c) Vicente, R. Org. Biomol. Chem. 2011, 9, 6469

[7] (a) Schultz, D. M.; Wolfe, J. P. Org. Lett. 2010, 12, 1028.

(b) Caddick, S.; Koe, W. Tetrahedron Lett. 2002, 43, 9347.

(c) Sharma, V.; Kumar, P.; Pathak, D. J. Heterocycl. Chem. 2010 47, 491

(d) Lu, T.; Lu, Z.; Ma, Z. X.; Zhang, Y.; Hsung, R. P. Chem. Rev. 2013, 113, 4862 .

[8] Cho, C. S.; Kim, J. S.; Oh, B. H. Tetrahedron 2000, 56, 7747.

[9] Cui, X. M. Chlor-Alkali Ind. 2000, 5, 172192 (in Chinese). (崔小明，氯碱工业, 2000, 5, 17219.)

[10] (a) Müller, T. E.; Hultzsch, K. C.; Yus, M.; Foubelo, F.; Tada, M. Chem. Rev. 2008, 108, 3795.

(b) Lu, Z.; Ma, S. M. Angew. Chem., Int. Ed. 2008, 47, 258.

(c) Luo, J.; Jiang, H. F. Chin. J. Org. Chem. 2008, 28, 187 (in Chinese).

(罗洁, 江焕峰, 有机化学, 2008, 28, 187.)

(d) Li, Y.; Zheng, Y.; Tian, F.; Zhang, Y. J.; Zhang, W. Chin. J. Org. Chem. 2009, 29, 1487 (in Chinese)

(李亚胥, 郑玉林，田丰涛，张勇健，张万斌，有机化学，2009, 29, 1487.)

(e) Lee, O. Y.; Law, K. L.; Yang, D. Org. Lett. 2009, 11, 3302.

(f) Huang, L.; Arndt, M.; Gooßen, K.; Heydt, H.; Gooßen, L. J. Chem. Rev. 2015, 115, 2596.

(g) Butt, N. A.; Zhang, W. Chem. Soc. Rev. 2015, 44, 7929.

[11] Lee, O. Y.; Law, K. L.; Yang, D. Org. Lett. 2009, 11, 3302.

[12] (a) Patel, S. J.; Jamison, T. F. Angew. Chem., Int. Ed. 2003, 42 , 1364.

(b) Patel, S. J.; Jamison, T. F. Angew. Chem., Int. Ed. 2004, 43, 3941.

(c) Zhou, C. Y.; Zhu, S. F.; Wang, L. X., Zhou, Q. L. J. Am. Chem. Soc. 2010, 132, 10955 .

(d) Holmes, M.; Schwartz, L. A.; Krische, M. J. Chem. Rev. 2018, 118,6026 .

[13] (a) Xie, Y. J.; Hu, J. H.; Wang, Y. Y.; Xia, C. G.; Huang, H. J. Am 
Chem. Soc. 2012, 134, 20613.

(b) Liu, Y.; Xie, Y.; Wang, H.; Huang, H. J. Am. Chem. Soc. 2016, $138,4314$.

[14] (a) Bäckvall, J. E.; Nordberg, R. E.; Nyström, J. E.; Hoegberg, T.; Ulff, B. J. Org. Chem. 1981, 46, 3479.

(b) You, S. L.; Zhu, X. Z.; Luo, Y. M.; Hou, X. L.; Dai, L. X. J. Am. Chem. Soc. 2001, 123, 7471 .

(c) Nagano, T.; Kobayashi, S. J. Am. Chem. Soc. 2009, 131, 4200.

(d) Xie, Y. J.; Hu, J. H.; Wang, Y. Y.; Xia, C.; Huang, H. J. Am. Chem. Soc. 2012, 134, 20613.

(e) Dubovyk, I.; Watson, I. D. G.; Yudin, A. K. J. Org. Chem. 2013, $78,1559$.

(f) Cai, A. J.; Guo, W. S.; Martínez-Rodríguez, L.; Kleij, A. W. J. Am. Chem. Soc. 2016, 138, 14194. (g) Li, Y. G.; Li, L.; Yang, M. Y.; Kantchev, E. A. B. J. Org. Chem. 2017, 82, 4907.

[15] (a) Trost, B. M.; Zhang, T.; Sieber, J. D. Chem. Sci. 2010, 1, 427.

(b) Evans, P.; Grange, R.; Clizbe, E. Synthesis 2016, 48, 2911.

(c) Guo, W.; Cai, A.; Xie, J.; Kleij, A. W. Angew. Chem., Int. Ed. 2017, 56, 11797 .

(d) Xia, C.; Shen, J.; Liu, D.; Zhang, W. Org. Lett. 2017, 19, 4251

(e) Wang, Y. N.; Wang, B. C.; Zhang, M. M.; Gao, X. W.; Li, T. R.; Lu, L. Q.; Xiao, W. J. Org. Lett. 2017, 19, 4094.

[16] Yao, L.; Wang, C. J. Adv. Synth. Catal. 2015, 357, 384.

[17] Lu, B.; Feng, B.; Ye, H.; Chen, J. R.; Xiao, W. J. Org. Lett. 2018, $20,3473$.

[18] Ouyang, K.; Xi, Z. Acta Chim. Sinica 2013, 71, 13 (in Chinese). (欧阳昆冰, 席振峰, 化学学报, 2013, 71, 13.)

(Zhao, C.) 